\title{
NONEXISTENCE OF SOME NONPARAMETRIC SURFACES OF PRESCRIBED MEAN CURVATURE
}

\author{
KIRK E. LANCASTER
}

\begin{abstract}
If $\Omega \subset \mathbf{R}^{2}$ has a reentrant corner, the Dirichlet problem for the equation of prescribed mean curvature $H$ with zero boundary value has no solution when $H$ has constant nonzero sign.
\end{abstract}

Suppose $\Omega$ is a bounded open subset of $\mathbf{R}^{n}, \phi \in C^{0}(\partial \Omega)$, and $H \in C^{0}(\Omega)$. Does there exist a solution $u \in C^{0}(\bar{\Omega}) \cap C^{2}(\Omega)$ of the Dirichlet problem

$$
\begin{aligned}
\operatorname{div}(T u) & =n H \quad \text { on } \Omega, \\
u & =\phi \quad \text { on } \partial \Omega,
\end{aligned}
$$

where $T u=\nabla u / W$ and $W=\sqrt{1+|\nabla u|^{2}}$ ? Using barrier arguments, Graham Williams [5] has proven this

THEOREM. Given $n \geqslant 2, K \in(0,1 / \sqrt{n-1}), R>0$, and $\Omega$ with locally Lipschitz boundary satisfying a uniform exterior sphere condition of radius $R$, for $\|H\|_{\infty}$ small enough there exists $\varepsilon>0$ depending on $n, K, R$, and $\|H\|_{\infty}$ such that whenever $\phi \in C^{0,1}(\partial \Omega)$ has Lipschitz constant $\leqslant K$ and $\sup \phi-\inf \phi<\varepsilon$, there exists $u \in$ $C^{0}(\bar{\Omega}) \cap C^{2}(\Omega)$ which satisfies $(1)$.

It has been known for some time that if the data is small and $\partial \Omega$ is smooth, (1) has a (classical) solution [4].

We will say that $\Omega \subset \mathbf{R}^{2}$ has a reentrant corner at $P \in \partial \Omega$ if for some $a, \alpha, \beta$ with $\beta-\alpha>\pi$ and $a>0,\{(r, \theta) \mid 0<r<a, \alpha \leqslant \theta \leqslant \beta\} \subset \Omega$, where $(r, \theta)$ represents polar coordinates about $P$. The purpose of this note is to prove the following

THEOREM. Let $n=2$ and $\Omega$ be a bounded open subset of $\mathbf{R}^{2}$ which has reentrant corner at $P \in \partial \Omega$. Suppose $H \in C^{0}(\Omega)$ with $H<0$ and $\phi \in C^{0}(\partial \Omega)$ with $\phi(P)=0$ and $\phi \geqslant 0$. Then there is no solution $u \in C^{0}(\bar{\Omega}) \cap C^{2}(\Omega)$ of $(1)$.

Using the results of [1], we can determine the behavior of the variational solution of (1) near $P$ when $\partial \Omega \backslash\{P\}$ has positive curvature. The case $n=2$ is interesting because of its relationship to the "membrane analogy" of engineering, which we discuss later.

Proof. Suppose a solution $u$ of (1) exists. We may assume $P$ is the origin and $-3 \pi / 4<\alpha=-\beta$. Let $V$ be the interior of the nonconvex quadrilateral symmetric with respect to the $x$-axis determined by the points $(r, \theta)=(0,0),(a, \beta),(a,-\beta)$,

Received by the editors April 15, 1985.

1980 Mathematics Subject Classification. Primary 35J25, 35J60.

(c) 1986 American Mathematical Society $0002-9939 / 86 \$ 1.00+\$ .25$ per page 
$(a, 0)$. Set $m=\inf \{u(r, \theta) \mid r=a / 4,-\beta \leqslant \theta \leqslant \beta\}$. By the strict maximum principle, $u>0$ on $\Omega$ and so $m>0$.

Define $k \in C^{0}(\partial V)$ by setting $k=0$ on $\partial V \cap S$, where $S=\{(r, \theta) \mid r<a / 2\}$, and $k=m$ on the two sides of $\partial V$ not touching $P$ and by requiring $k$ to be linear on each of the remaining portions of $\partial \Omega$.

Now let $f$ be the variational solution of the Dirichlet problem for the minimal surface equation in $V$ with boundary values $k$. Then $f \in C^{2}(V) \cap C^{0}(\bar{V} \backslash\{P\})$ and $f=k$ on $\partial V \backslash\{P\}$. Notice $0 \leqslant f \leqslant m$ and $u \geqslant m$ on $r=a / 4,-\beta \leqslant \theta \leqslant \beta$, so $f \leqslant u$ on $r=a / 4,-\beta \leqslant \theta \leqslant \beta$. Since $u \geqslant 0$ and $k=0$ on $\partial V \cap S$, we see that $0 \leqslant f \leqslant u$ in $V \cap\{(r, \theta) \mid r<a / 4\}$. This implies $f \in C^{0}(\bar{V})$, in contradiction to [2, pp. 146-147]. Q.E.D.

Suppose $\Omega$ is connected and simply connected in $\mathbf{R}^{2}$. Consider a uniform bar with cross section $\Omega$. If we apply couples to the ends and twist the bar, then the stress function $g$ satisfies

$$
\Delta g=2 H \text { on } \Omega, \quad g=0 \text { on } \partial \Omega,
$$

where $H=-G A, G$ is the modulus of rigidity of the bar, and $A$ is the angle of twist per unit length of the bar. The membrane analogy is the assumption that $g \approx u$ and $\nabla g \approx \nabla u$ on $\Omega$, where $g$ solves (2) and $u$ solves (1) with $n=2$ and $\phi \equiv 0$. If $\Omega$ has a reentrant corner, no solution of (1) exists and the analogy is invalid. For a discussion of the membrane analogy and some experiments it inspired, see [3].

Acknowledgement. I wish to thank Professor Gulliver for his comments and encouragement.

\section{REFERENCES}

1. A. Elcrat and K. Lancaster, Boundary behavior of a non-parametric surface of prescribed mean curvature near a reentrant corner (to appear).

2. R. Finn, Remarks relevant to minimal surfaces, and to surfaces of prescribed mean curvature, J. Analyse Math. 14 (1965), 139-160.

3. T. Higgins, Analogic experimental methods in stress analysis as exemplified by Saint-Venant's torsion problem, Experimental Stress Analysis 2 (1945), 17-27.

4. R. Gulliver, personal communication.

5. G. Williams, The Dirichlet problem for the minimal surface equation with Lipschitz continuous boundary data, Research Report CMA-R41-83, Australian National Univ., 1983.

Department of Mathematics and Statistics, Wichita State University, Wichita, Kansas 67208 